\title{
Correlation of dysphagia with functional decline in neurodegenerative disease - a review
}

\begin{abstract}
Hypothesis: The functional decline noted over time in patients with neuromuscular diseases is associated with increasing risk of dysphagia and aspiration.

Study design: Systematic literature review.

Methods:A Pub Med search to identify all of the literature addressing neuromuscular disease progression and correlation with dysphagia was performed in the English literature from $1 / 1 / 2000$ through 4/1/2015. Studies with outcomes measures for both functional decline and dysphagia or aspiration were included, whereas stroke and head injury were excluded.

Results: Of the 309 articles identified in the search, 13 discussing Parkinson's disease PD and 5 on the topic of Amyotrophic Lateral Sclerosis ALS met the criteria. No papers on the subjects of Multiple Sclerosis MS or Huntington's disease HD were identified. Articles were stratified by level of evidence using the criteria of Sackett et al., ${ }^{1}$ noting instrument selection for disease state and dysphagia, self-reported vs. observed, outcome and limitations. There were 8 true longitudinal studies, and the other 10 stratified patients by duration or disease severity. Overall, the studies support increased dysphagia or swallowing related quality of life decline in patients with advanced PD and ALS. However, most studies are limited by 1) the cross-sectional nature instead of true longitudinal design, 2) self-reported instruments and 3) infrequent mention of aspiration risk.
\end{abstract}

Conclusion:Progressive dysphagia and aspiration risk is weakly demonstrated with advancing PD and ALS. Although these are similar known risks with MS and HD, there are only anecdotal reports. A systematic approach at screening these patients for dysphagia is warranted.

Keywords: dysphagia, neurodegenerative disease, als, huntington's disease, parkinson's disease
Volume 7 Issue 4 - 2017

\author{
Kenneth W Altman, Felicia L Carter ,Valerie \\ Peicher \\ Department of Otolaryngology Head Neck Surgery Baylor \\ College of Medicine Houston, USA
}

Correspondence: Kenneth W Altman Department of Otolaryngology Head Neck Surgery Baylor College of Medicine Houston, USA, Tel 713-798-5900,

Email Kenneth.altman@bcm.edu

Received: March 26, 2017 | Published: June 13, 2017
Abbreviations: PD, parkinson's disease; ALS, amyotrophic lateral sclerosis; MS, multiple sclerosis; MBSS, modified barium swallow study; MRC, medical research council; ALSFRS, als functional rating system

\section{Introduction}

Neurodegenerative diseases including Parkinson's disease PD, Amyotrophic Lateral Sclerosis ALS, Multiple Sclerosis MS, and Huntington's disease HD are characterized by a progressive decline in functional status. ${ }^{2-20}$ The progression of dysphagia in neurodegenerative disease has been well described by Hadjikoutis et al., ${ }^{21}$ with ALS. The voluntary swallow is affected first; initially the swallow reflex is delayed and eventually it degenerates entirely. Spontaneous reflexive swallows are lost typically in the pre-terminal stage of the disease. Malnutrition becomes an issue, which in turn worsens coordination and strength of the deglutition muscles, as well as muscles required for respiratory function. As the disease progresses, oral hygiene often deteriorates as it is more difficult for patients to perform self-care such as teeth brushing, as well as difficulty clearing their oral secretions. Thus food particles remain and can increase oral bacterial load, increasing the risk of aspiration pneumonia. ${ }^{21}$

Dysphagia often goes unnoticed until a catastrophic event occurs, such as an aspiration event or aspiration pneumonia. Catastrophic events result in negative outcomes for the patient, including inability to take needed medicines, prolonged hospitalizations, deconditioning, and malnutrition. ${ }^{22}$ The debilitating natural progression of neurodegenerative disease introduces an added degree of difficulty to recover from such events. Patients, families and care teams are then faced with the decision to pursue alternative means of nutrition and hydration given the severity of dysphagia. Thus it is highly beneficial to patients, their families, and health care systems to have established protocols to screen patients at high aspiration risk for dysphagia. ${ }^{22}$ In addition to the life-threatening consequences of dysphagia and aspiration with neuromuscular disease, quality of life is threatened as eating becomes a stressful event rather than a pleasurable experience. ${ }^{5}$ The social stresses are severe as meal times are quintessential to personal bonding and professional networking. ${ }^{23}$

For all of these reasons, it is vital to develop a standard practice to detect dysphagia in patients with neurodegenerative disease early on, when dietary changes and behavioral training can extend quality of life. ${ }^{12-21}$ Thus we sought to identify and quantify relationships between disease progression and dysphagia progression and/or aspiration risk in patients with neurodegenerative disease through a systematic review of the existing literature. Our hypothesis is that overall functional decline in neuromuscular disease over time is associated with worsening dysphagia and aspiration risk.

\section{Methods}

A Pub Med search over the time period 1/1/2000 through 4/1/2015 was performed for the following terms: neuromuscular disease or 
Parkinson's or PD or ALS or amyotrophic lateral sclerosis or HD or Huntington's disease or Huntington's chorea or MS or multiple sclerosis or movement disorder AND functional status or functional decline or decline or activities of daily living or ADL or quality of life or QOL AND dysphagia or swallowing or aspiration or malnutrition or malnourishment. The inclusion criteria were that an article must:

I. Address a neuromuscular disease (PD, ALS, HD, MS) in regards to functional status or quality of life and dysphagia, and

II. Must be written in English. The exclusion criteria were:

a. studies published before 2000 ,

b. articles not available in full text,

III. Basic science articles,

IV. Animal models,

V. Review articles, and

VI. Subjects primarily or confounded by stroke or head injury.
Selected articles were tabulated and compared with regard to level of evidence, number of subjects, follow up period, objective and subjective measures used to assess functional status, and measure of dysphagia.

\section{Results}

The initial search yielded 309 articles. After applying inclusion and exclusion criteria, 18 articles remained (see process in PRISMA diagram in Figure 1. Table 1 describes the instruments used to assess the extent of the neuromuscular diseases encountered in the course of the review, including information source and limitations. Table 2 does the same for instruments used to measure dysphagia relative to this review. Of the 18 articles available for qualitative review, 13 were specific for PD, and 5 were specific for ALS. The search did not yield any papers that studied the progression of Multiple Sclerosis or Huntington's disease over time with respect to functional status or dysphagia. Table 3 summarizes the findings of the 18 papers reviewed in this study. A formal meta-analysis was not able to be performed based on the spectrum of study criteria.

Table I Functional status instruments in neuromuscular diseases

\begin{tabular}{|c|c|c|c|c|}
\hline $\begin{array}{l}\text { Neuromuscular } \\
\text { Disease Instrument }\end{array}$ & $\begin{array}{l}\text { Self-Reported Vs. } \\
\text { Observed Vs. Measured }\end{array}$ & Reference/Yr & Description & Limitation \\
\hline $\begin{array}{l}\text { PD Questionnaire (PDQ) } \\
39\end{array}$ & Self-reported & Walker et al. ${ }^{4}$ & $\begin{array}{l}39 \text { questions rating no difficulty } \\
\text { with ADLs }(0) \text { to always have } \\
\text { difficulty }(100) \text {. }\end{array}$ & Subjective \\
\hline $\begin{array}{l}\text { I } 5 \text { item Geriatric } \\
\text { Depression Scale }\end{array}$ & Self-reported & Miller \& Weintraub et al. ${ }^{9,36}$ & $\begin{array}{l}30 \text { yes/no questions rating no } \\
\text { depression }(\leq 5) \text { to severe } \\
\text { depression }(=15) \text {. }\end{array}$ & Subjective \\
\hline Hoehn \& Yahr (HY) scale & Observed & Carneiro \& Hoehn et al.,20 & $\begin{array}{l}\text { Stages } I-V \text { ranging from no } \\
\text { functional impairment }(I) \text { to inability } \\
\text { to ambulate independently }(\mathrm{V}) \text {. }\end{array}$ & $\begin{array}{l}\text { No half stages. } \\
\text { Progression not } \\
\text { necessarily linear. }\end{array}$ \\
\hline $\begin{array}{l}\text { ALS-Functional Rating } \\
\text { Score (FRS) }\end{array}$ & Self-reported & Voustianiouk \& Magnus et al. ${ }^{18,33}$ & $\begin{array}{l}\text { Rating lowest functioning }(0) \text { to } \\
\text { normal }(40) \text { based on } 10 \text { question } \\
\text { survey. }\end{array}$ & $\begin{array}{l}\text { Does not weigh } \\
\text { respiratory dysfunction } \\
\text { the same as limb/bulbar } \\
\text { function } 32\end{array}$ \\
\hline ALSFRS-R & Observed or self-reported & Cedarbaum \& Nakamura et al. ${ }^{16,35}$ & $\begin{array}{l}\text { Health care provider determines } \\
\text { functional status rating lowest } \\
\text { functioning }(0) \text { to normal } \\
\text { functioning }(48) \text {. }\end{array}$ & $\begin{array}{l}\text { Lacking correlation } \\
\text { between FVC\% and } \\
\text { respiratory subscale } 32\end{array}$ \\
\hline $\begin{array}{l}\text { Medical Research Council } \\
\text { (MRC) for neck flexors }\end{array}$ & Observed & Nakamura et al. ${ }^{16}$ & Full clinical exams by neurologists. & Rater-dependent \\
\hline $\begin{array}{l}\text { Medical Research Council } \\
\text { (MRC) scale }\end{array}$ & Observed & Chiò et al. ${ }^{18}$ & $\begin{array}{l}\text { Scored 0-5. Tests neck flexors \& } \\
\text { extensors, and upper \& lower limb } \\
\text { muscles }\end{array}$ & \\
\hline $\begin{array}{l}\text { Medical Research Council } \\
\text { (MRC) Compound Score }\end{array}$ & Self-reported & Magnus et al. ${ }^{17}$ & 9-step modified MRC scale & \\
\hline $\begin{array}{l}\text { Percent Predicted Forced } \\
\text { Vital Capacity (FVC) \% }\end{array}$ & Measured & Magnus et al. ${ }^{17}$ & Assess lung function & $\begin{array}{l}\text { Dependent on subject's } \\
\text { effort }\end{array}$ \\
\hline $\begin{array}{l}\text { Mini Mental State Exam } \\
\text { (MMSE) }\end{array}$ & Observed & Walker et al. ${ }^{4}$ & $\begin{array}{l}\text { Score } 0-30 \\
<22=\text { cognitive impairment, may } \\
\text { need caretaker to answer questions }\end{array}$ & $\begin{array}{l}\text { Weakly detects mild } \\
\text { cognitive impairment }\end{array}$ \\
\hline $\begin{array}{l}\text { Schwab and England } \\
\text { Activities of Daily Living } \\
\text { (ADL) }\end{array}$ & Self-reported or observed & Auyeung et al. "' & $\begin{array}{l}\text { Bed-ridden ( } 0) \text { to independent } \\
(100) . \leq 30=\text { dependent. } \geq 80= \\
\text { independent. Measured drug "on". }\end{array}$ & Subjective \\
\hline $\begin{array}{l}\text { Beck Depression } \\
\text { Inventory (BDI) }\end{array}$ & Self-reported & Plowman-Prine et al. ${ }^{6}$ & $\begin{array}{l}21 \text { domains rating depression: none } \\
(0) \text { to most severe }(63) \text {. }\end{array}$ & Subjective \\
\hline $\begin{array}{l}\text { Non-motor symptom } \\
\text { scale }\end{array}$ & Self-reported & Guo et al. ${ }^{3}$ & Assess severity/ frequency of NMS & Subjective \\
\hline ALS severity score & Self-reported & del Aguila et al. ${ }^{19}$ & $\begin{array}{l}\text { Self-reported performance in } \\
\text { speech, swallow, lower \& upper } \\
\text { extremity function rating worst }(0) \\
\text { to best }(40) \text {. }\end{array}$ & Subjective \\
\hline
\end{tabular}


Table Continued

\begin{tabular}{|c|c|c|c|c|}
\hline $\begin{array}{l}\text { Neuromuscular } \\
\text { Disease Instrument }\end{array}$ & $\begin{array}{l}\text { Self-Reported Vs. } \\
\text { Observed Vs. Measured }\end{array}$ & Reference/Yr & Description & Limitation \\
\hline $\begin{array}{l}\text { El Escorial Diagnostic } \\
\text { Criteria (EEDC) }\end{array}$ & Observed & Chiò et al. ${ }^{18}$ & $\begin{array}{l}\text { Standard tool to assess ALS status. } \\
\text { Includes signs of progressive } \\
\text { upper and lower motor neuron } \\
\text { degeneration }\end{array}$ & \\
\hline $\begin{array}{l}\text { Unified PD Rating Scale } \\
\text { (UPDRS) }\end{array}$ & $\begin{array}{l}\text { Self-report }+ \text { clinical } \\
\text { observation }\end{array}$ & Carneiro \& Ramaker et al. ${ }^{2,26}$ & $\begin{array}{l}\text { Part I: evaluate mentation, mood, } \\
\text { behavior } \\
\text { Part II: self-eval ADLs } \\
\text { Part III: clinician-scored monitored } \\
\text { motor eval } \\
\text { Part IV: HY staging } \\
\text { Part V: Schwab and England ADL } \\
\text { scale }\end{array}$ & $\begin{array}{l}\text { Redundancy with } \\
\text { several items focusing } \\
\text { on the same construct }\end{array}$ \\
\hline $\begin{array}{l}\text { Hospital Anxiety and } \\
\text { Depression (HAD) scale }\end{array}$ & Self-reported & Walker \& Zigmond et al. ${ }^{4,30}$ & $\begin{array}{l}\text { Questions assessing anxiety and } \\
\text { depression rating from } 0-2 \mathrm{I},>8= \\
\text { abnormal }\end{array}$ & Subjective \\
\hline $\begin{array}{l}\text { Frontal Assessment } \\
\text { Battery (FAB) }\end{array}$ & Observed & Antonini \& Dubois et al. ${ }^{10,31}$ & $\begin{array}{l}\text { Bedside test of executive } \\
\text { functioning [28] }\end{array}$ & \\
\hline $\begin{array}{l}\text { Hamilton Depression } \\
\text { Scale (HAM-D) }\end{array}$ & Self-reported & Antonini \& Hamilton et al. ${ }^{10,32}$ & $\begin{array}{l}\text { I7 variables rating depression from } \\
\text { absent }(0) \text { to severe }(4)\end{array}$ & $\begin{array}{l}\text { Interviewer dependent. } \\
\text { Only for patients with } \\
\text { diagnosed depressive } \\
\text { disorder [28] }\end{array}$ \\
\hline $\begin{array}{l}\text { Health-related QOL using } \\
\text { the Medical Outcomes } \\
\text { Study 36-Item Short-Form } \\
\text { General Health Surveyl6, } \\
\text { and Schedule for the } \\
\text { Evaluation of Individual } \\
\text { QOL-Direct Weighting } \\
\text { [15] }\end{array}$ & Self-reported & Speyer28 & $\begin{array}{l}\text { Dysphagia's impact on quality of life } \\
\text { as per the patient }\end{array}$ & Subjective \\
\hline Norris ALS disability scale & Observed & Chio et al. ${ }^{18}$ & $\begin{array}{l}\text { Tracks change in impairments } \\
\text { \& disability measures after ALS } \\
\text { treatment }\end{array}$ & \\
\hline Appel Rating Scale & Observed & Chio \& Appel et al. ${ }^{18,34}$ & $\begin{array}{l}\text { Assess swallow, speech, and } \\
\text { respiratory function rating normal } \\
\text { (30) to maximum dysfunction (164) }\end{array}$ & \\
\hline $\begin{array}{l}\text { Phonation time as } \\
\text { reflection of vital capacity }\end{array}$ & Observed & del Aguila et al. ${ }^{19}$ & $\begin{array}{l}\text { Completed } 3 \text { times, longest trial } \\
\text { analyzed }\end{array}$ & \\
\hline
\end{tabular}

Table 2 Swallow assessment instruments encountered in our review pertaining to neuromuscular diseases

\begin{tabular}{|c|c|c|c|c|}
\hline Dysphagia Instrument & $\begin{array}{l}\text { Self-Reported Vs. } \\
\text { Observed Vs. Measured }\end{array}$ & Reference & Description & Limitation \\
\hline SWAL-QOL & Observed & McHorney et al..$^{25}$ & $\begin{array}{l}44 \text { questions that evaluate II domains of QOL: } \\
\text { burden, eating duration/desire, sx frequency, food } \\
\text { selection, communication, fear, mental health, social, } \\
\text { fatigue, sleep }\end{array}$ & Subjective \\
\hline $\begin{array}{l}\text { Swallow fnx assessed by } \\
\text { physician w/ 7-grade scale }\end{array}$ & Observed & Waxman et al. ${ }^{26}$ & $\begin{array}{l}\text { Dysphagia rating normal function }(0) \text { to severe } \\
\text { dysphagia (6) }\end{array}$ & \\
\hline Trial swallow using water & Observed & Speyer ${ }^{28}$ & $\begin{array}{l}\text { Patient swallows } 150 \mathrm{ml} \text { of water as quickly as } \\
\text { possible in "off drug" state }\end{array}$ & \\
\hline $\begin{array}{l}\text { Kennedy et al.'s Modified } \\
\text { dysphagic rating scale (mDRS) }\end{array}$ & Observed/self-reported & Kennedy et al. ${ }^{27}$ & I 2 items rating dysphagia worst $(I)$ to normal (5) & \\
\hline Speech/swallowing difficulty & Observed/self-reported & Auyeung et al." & Assessing for presence \& time to onset yearly & \\
\hline Electromyography (EMG) & Measured & Potulska et al. ${ }^{13}$ & $\begin{array}{l}\text { Electrodes taped over swallow muscles, then } \\
\text { patients swallow dry and I- } 20 \mathrm{ml} \text { of water }\end{array}$ & \\
\hline $\begin{array}{l}\text { Scintigraphy of esophageal } \\
\text { activity }\end{array}$ & Measured & Speyer ${ }^{28}$ & $\begin{array}{l}\text { Gamma camera tracks aspiration and bolus } \\
\text { movement; degree of reflux }\end{array}$ & \\
\hline $\begin{array}{l}\text { Modified barium swallow with } \\
\text { videofluoroscopy }\end{array}$ & Measured & Monte et al. ${ }^{14}$ & Quantifies swallow abnormalities & $\begin{array}{l}\text { Only captures a moment } \\
\text { of swallow function }\end{array}$ \\
\hline
\end{tabular}


Table 3 Patients with Parkinsonism were distributed as follows: 17 PD, 15 MSA, 14 Lewy body dementia, 13 Corticobasal degeneration, 24 progressive supranuclear pals

\begin{tabular}{|c|c|c|c|c|c|c|c|c|}
\hline Author & Year & Dz & LOE & $\begin{array}{l}\text { F/u Period, } \\
\text { Time Range }\end{array}$ & Subjects & Outcome Measures & Major Findings & Limitations \\
\hline $\begin{array}{l}\text { Carneiro } \\
\text { et al. }{ }^{2}\end{array}$ & 2014 & PD & 4 & I snap shot & 103 & $\begin{array}{l}\text { SWAL-QOL: } 44 \text { questions } \\
\text { that evaluate II domains of } \\
\text { QOL } \\
\text { HY: classification performed } \\
\text { by neurologist at Pro- } \\
\text { Parkinson program } \\
\text { UPDRS: self-report + clinical } \\
\text { observation }\end{array}$ & $\begin{array}{l}\text { HY stage progresses with PD } \\
\text { PD progression worsens swallowing } \\
\text { QOL, specifically for eating duration, } \\
\text { symptom frequency, and sleep. }\end{array}$ & $\begin{array}{l}\text { Dysphagia self- } \\
\text { reported, no objective } \\
\text { measure. }\end{array}$ \\
\hline Guo et al. ${ }^{3}$ & 2013 & PD & 4 & I snap shot & 616 & $\begin{array}{l}\text { Non-motor symptom scale: } \\
\text { assess severity/frequency } \\
\text { of NMS } \\
\text { HY for disease severity } \\
\text { Standard questionnaire on } \\
\text { dx delay, disease duration, } \\
\text { anti-PD meds } \\
\text { UPDRS part } 3 \text { to assess } \\
\text { motor disability }\end{array}$ & $\begin{array}{l}\text { Non-motor symptoms (swallowing) } \\
\text { increased with disease progression }\end{array}$ & $\begin{array}{l}\text { Cross sectional study } \\
\text { does not necessarily } \\
\text { show progression. }\end{array}$ \\
\hline $\begin{array}{l}\text { Walker et } \\
\text { al. }{ }^{4}\end{array}$ & 2011 & PD & 4 & I snap shot & 75 & $\begin{array}{l}\text { PDQuestionnaire-39: disease } \\
\text { duration } \\
\text { Mini-Mental State } \\
\text { Examination } \\
\text { Hospital Anxiety and } \\
\text { Depression scale } \\
\text { HY stages } \\
\text { UPDRS on meds }\end{array}$ & $\begin{array}{l}32 \% \text { of patients had dysphagia, which } \\
\text { correlated with quality of life but not } \\
\text { with disease duration }\end{array}$ & $\begin{array}{l}\text { Self reported } \\
\text { dysphagia, need } \\
\text { objective measure. }\end{array}$ \\
\hline $\begin{array}{l}\text { Leow et } \\
\text { al. }^{5}\end{array}$ & 2010 & PD & 4 & I snap shot & 68 & $\begin{array}{l}\text { HY Stages } \\
\text { SWAL-QOL "on" meds }\end{array}$ & $\begin{array}{l}\text { Disease progression decreases QOL. } \\
\text { Later-stage PD = decreased appetite, } \\
\text { difficulty } w / \text { food selection, prolonged } \\
\text { meal time, stress surrounding social } \\
\text { meals }\end{array}$ & $\begin{array}{l}\text { Results may be } \\
\text { influenced by } \\
\text { confounding variables } \\
\text { such as depression. }\end{array}$ \\
\hline $\begin{array}{l}\text { Plowman- } \\
\text { Prine et } \\
\text { al. }{ }^{6}\end{array}$ & 2009 & PD & 4 & I snap shot & 36 & $\begin{array}{l}\text { HY Stages } \\
\text { SWAL-QOL } \\
\text { PDQ-39, Beck Depression } \\
\text { Inventory } \\
\text { UPDRS "on" meds }\end{array}$ & $\begin{array}{l}\text { No relation between swallow-specific } \\
\text { QOL + disease duration /severity. } \\
\text { Dysphagia significantly reduces total } \\
\text { SWAL-QOL. }\end{array}$ & $\begin{array}{l}\text { Dysphagia vs. } \\
\text { nondysphagia patients } \\
\text { compared and then } \\
\text { checked for HY. Not a } \\
\text { longitudinal study. }\end{array}$ \\
\hline $\begin{array}{l}\text { Lorefält et } \\
\text { al. }{ }^{7}\end{array}$ & 2005 & PD & 2 & $\begin{array}{l}\text { I year at } 2 \text { time } \\
\text { points }\end{array}$ & 52 & $\begin{array}{l}\text { UPDRS } \\
\text { Swallow assessed by } \\
\text { physician with } 7 \text {-grade scale: } \\
\text { normal to severe dysphagia } \\
\text { as per Waxman et al. } 1990\end{array}$ & $\begin{array}{l}\text { No significant changes in dysphagia in } \\
\text { PD patients over I year }\end{array}$ & $\begin{array}{l}\text { PD may not have } \\
\text { progressed in that } \\
\text { time. }\end{array}$ \\
\hline $\begin{array}{l}\text { Müller et } \\
\text { al. }{ }^{8}\end{array}$ & 2001 & PD & 4 & $\begin{array}{l}\text { Retrospective } \\
\text { post-mortem }\end{array}$ & $83 *$ & $\begin{array}{l}\text { Patient-reported latencies to } \\
\text { dysphagia } \\
\text { National Institute of } \\
\text { Neurological Disorders and } \\
\text { Stroke for PSP dx } \\
\text { Neurologist recorded time } \\
\text { to speech disorder } \\
\text { Survival time }\end{array}$ & $\begin{array}{l}\text { Dysphagia stays latent for } \sim 84 \text { months } \\
\text { in PD, survival time post-dysphagia } \\
\text { complaint onset } \sim 15-24 \text { mo. Total } \\
\text { survival time correlated to latency to } \\
\text { complaint of dysphagia. }\end{array}$ & $\begin{array}{l}\text { Retrospective nature } \\
\text { precludes neurologists } \\
\text { evaluating patients } \\
\text { to use agreed upon } \\
\text { protocol. }\end{array}$ \\
\hline $\begin{array}{l}\text { Miller et } \\
\text { al. }{ }^{9}\end{array}$ & 2008 & PD & 4 & I snap shot & 137 & $\begin{array}{l}\text { Geriatric Depression Scale } \\
\text { HY stages } \\
\text { MMSE } \\
\text { Patients swallow } 150 \mathrm{ml} \text { of } \\
\text { water as quickly as possible } \\
\text { "off" meds } \\
\text { UPDRS "off" meds }\end{array}$ & $\begin{array}{l}\text { Moderate correlation between } \\
\text { swallowing rate and disease severity, } \\
\text { but not between swallowing speed } \\
\text { and disease duration. Self-report is not } \\
\text { reliable. Need objective assessment of } \\
\text { aspiration risk to compare with water } \\
\text { swallow test performance }\end{array}$ & $\begin{array}{l}\text { Did not follow } \\
\text { patients over time. }\end{array}$ \\
\hline $\begin{array}{l}\text { Antonini et } \\
\text { al. }{ }^{10}\end{array}$ & 2012 & PD & 2 & 2 years & 707 & $\begin{array}{l}\text { PDQ-39 } \\
\text { HY } \\
\text { MMSE } \\
\text { Frontal Assessment Battery } \\
\text { Hamilton Depression Scale } \\
\text { UPDRS part III }\end{array}$ & $\begin{array}{l}\text { Gl symptoms (dysphagia) worsened } \\
\text { + became more prevalent over } 2 \\
\text { years. Non-motor symptom progress } \\
\text { is symptom specific, does not follow } \\
\text { motor deterioration. Only some } \\
\text { symptoms negatively impact QOL }\end{array}$ & $\begin{array}{l}\text { Didn't assess } \\
\text { NMS severity, only } \\
\text { presence/ absence. }\end{array}$ \\
\hline
\end{tabular}


Table Continued

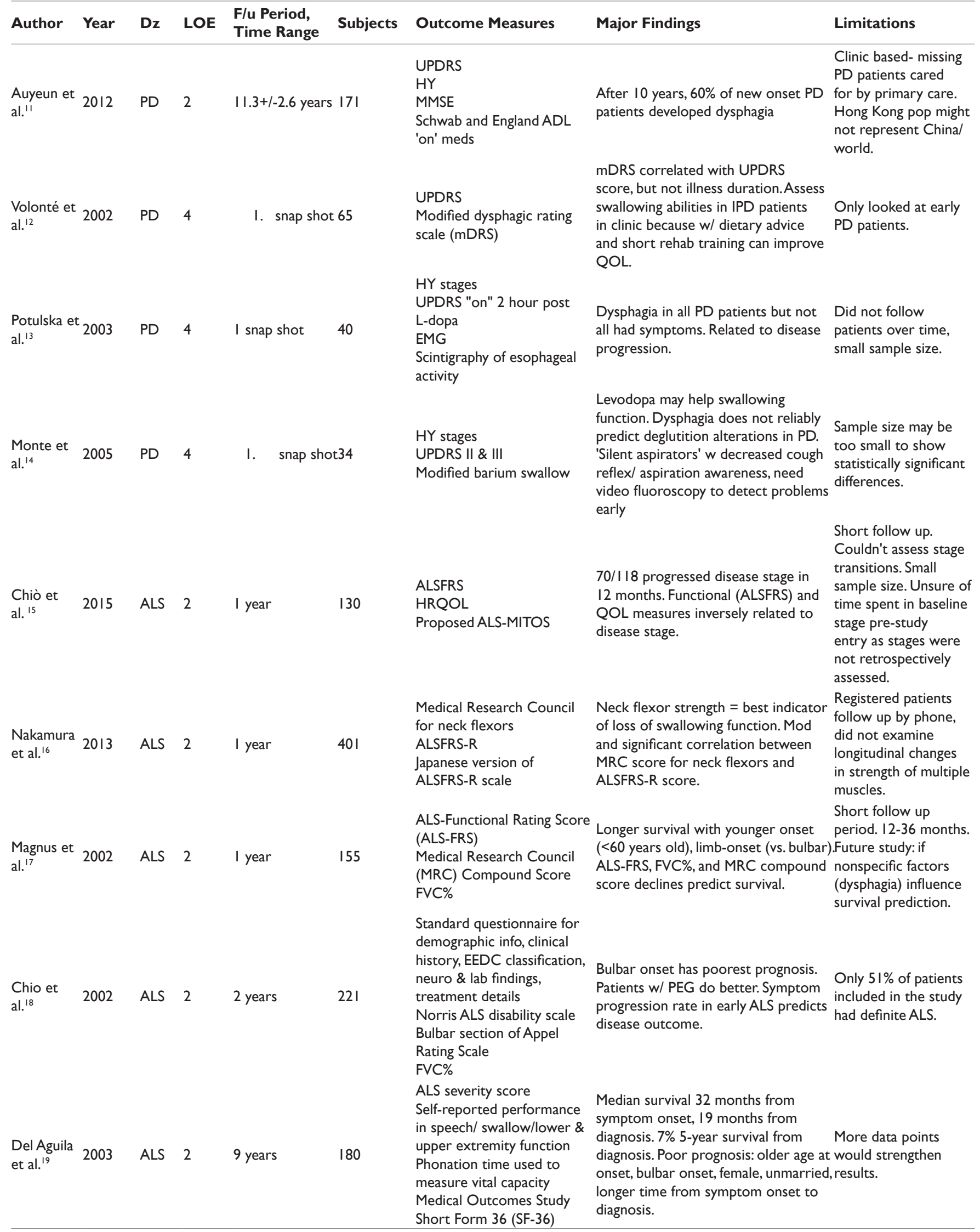

Abbreviations: ALS: amyotrophic lateral sclerosis; Dz: Disease; HY: Hoehn and Yahr; LOE: Level of Evidence; MMSE: Mini Mental State Exam; PD: Parkinson's disease; QOL: Quality of Life 


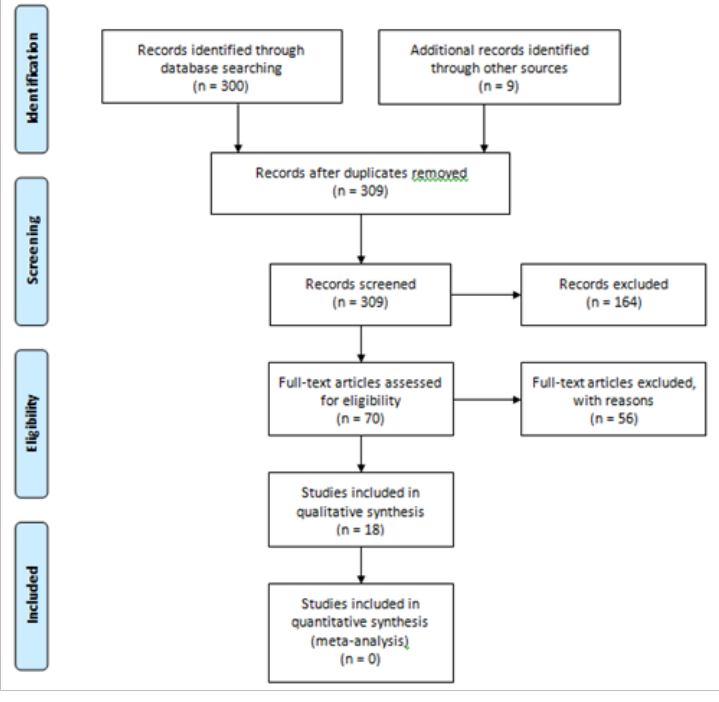

Figure I PRISMA Flow Diagram.

Nine of the articles that addressed dysphagia in PD studied patients at one point in time, and compared subjects based on Hoehn and Yahr (HY) stage ( 5 studies 2-14), disease duration 1 study, ${ }^{3}$ both HY stage and disease duration 2 studies, ${ }^{4,9}$ or just at time of study enrollment early in PD disease 1 study. ${ }^{12}$ Three of the studies followed patients every few months for 1-10 years. ${ }^{7-11}$ One study was retrospectively done by reviewing charts post-mortem. ${ }^{8}$ The subject count ranged from 34 to 707 people.

Six articles found that dysphagia is worse at more advanced stages of Parkinson's disease. ${ }^{2-13}$ One article that followed patients over the course of 2 years found that gastroenterology GI disturbances (dysphagia) became more prevalent in this period, but it was not directly linked to disease progression necessarily. ${ }^{10} \mathrm{An}$ article that followed patients with Parkinson's disease for 10 years found that at the end of the time period, $60 \%$ of patients had developed dysphagia, but it did not comment specifically on the development of dysphagia with regards to disease progression. ${ }^{11}$ Two articles found no correlation between dysphagia and disease duration. ${ }^{4-6}$ One article that followed patients over the course of a year found no significant changes in dysphagia, but there may have not been any significant changes in disease progression. ${ }^{7}$

The search also yielded 5 studies on disease progression in ALS. ${ }^{15,16}$ Chio et al., ${ }^{15}$ showed that the majority of patients progressed to a higher stage of disease in 12 months as quantified by the ALS Functional Rating System (ALSFRS) and by Quality of Life (QOL) measures. Nakamura et al., ${ }^{16}$ used the Medical Research Council (MRC) score to rate neck flexor muscle strength in relation to ALS disease progression. They found that neck flexor strength is the most significant prognostic factor for loss of swallowing function and that MRC score is significantly correlated to ALSFRS-R score. ${ }^{16}$ An additional 3 studies on ALS progression were included which did not have dysphagia-specific outcomes measures beyond those included in general self-reporting or those measures included in the ALS inventories. These studies show that onset of ALS is predictive of survival, specifically younger age and limb instead of bulbar involvement at onset predicts longer lifespan. ${ }^{17-19}$ Additional protective factors include being male, married, and diagnosed quickly after symptom onset. ${ }^{18}$ Chiò et al. ${ }^{18}$ found that PEG tube insertion often results in longer survival. ${ }^{18}$ Magnus et al., ${ }^{17}$ found that ALS-
FRS, functional vital capacity (FVC\%), and MRC compound score were predictive of survival. All five studies on ALS followed patients for no more than 2 years.

\section{Discussion}

It is intuitive to expect that as patients with neuromuscular and neurodegenerative disease have declining functional status, they also have a concomitant decline in the quality of their swallow mechanism with increased aspiration risk. Although there is significantly different pathophysiology with the neuromuscular diseases addressed in our review, common manifestations include the progressive nature of decline, increasing sedentary behavior with reduced respiratory function and pulmonary toilet, the increasing prevalence of dysphagia, and aspiration as a common precipitating terminal event. Our review reveals some correlation between functional decline and dysphagia with some neurodegenerative diseases (PD and ALS), however a quantifiable association and true longitudinal nature of this relationship was not able to be assessed. ${ }^{4-9}$ Functional status and dysphagia are both difficult to objectively measure in patients with neurodegenerative disease for a wide variety of reasons. Methods or surveys (instruments) can be categorized as self-reported vs. observed, which each carry with them their own unique set of limitations. Self-reporting (subjective instruments) is not fully reliable as a means of identifying dysphagia, or even functional status with neurodegenerative disease. ${ }^{9}$ There may be cognitive impairments associated with the neurodegenerative disease that may impact selfreporting. Studies have shown inconsistencies between patients' actual swallowing abilities and perceived difficulties. ${ }^{9-14}$ Additionally, patients may be fearful of losing their independence and pleasure in eating if they reveal symptoms of possible aspiration. ${ }^{5}$ There is also variability in the instrument reported by the patient versus a close family member.

Observation is more objective and scientifically controlled, but usually only captures one snapshot of time. ${ }^{14}$ This is a major limitation to fully understanding the extent of neurodegenerative diseases as their effects fluctuate based on time of day and medication dosage. Observing patients in drug "on" vs. "off" states as with PD may yield vastly different data points. Furthermore, the concepts of inter-rater and intra-rater variability are similar limitations with such a scale. In addition to clinician-observed scales, more objective information on functional status and dysphagia may be garnered from objective diagnostic testing, such as modified Barium swallow study (MBSS) to detect dysphagia and aspiration based on varying food consistencies and compensatory swallow maneuvers. ${ }^{24}$ These studies may show progression that may not yet be clinically relevant. ${ }^{8}$

There is a great need for consistent clinical use of a meaningful dysphagia rating scale early in the neurodegenerative disease process in order to improve patient and caregiver education on the importance of maintaining nutrition while realizing aspiration risk. ${ }^{4}$ More objective measures with longitudinal studied in the literature are necessary to paint an accurate clinical picture of patients' swallowing ability to recognize "sentinel indicators of decline" to reduce likelihood of dangerous complications including aspiration and pneumonia..$^{2-14}$ Detecting dysphagia early is also important because patients can undergo dietary changes and rehabilitation that can significantly improve their quality of life. ${ }^{12}$ One post-mortem study on PD patients documented longer total survival time when there was a longer latency period between clinically assessed initial dysphagia development and patient awareness of its complications. ${ }^{8}$ Therefore, by identifying a stronger correlation between functional decline in these diseases and dysphagia with aspiration risk, clinicians may more 
accurately predict when swallowing intervention would proactively be needed. ${ }^{25-30}$

None of the studies identified in our review were able to determine if dysphagia is a prognostic indicator of survival. Therefore, there is also a great need for a prospective study to follow a cohort of these patients and determine the effects of dysphagia on survival outcomes. Longitudinal studies to better define the devastating natural history of the neuromuscular diseases such as those associated with dysphagia would further empower care teams in order to better meet patient needs to enhance quality of life..$^{30-36}$

\section{Conclusion}

Neuromuscular and neurodegenerative diseases are often associated with dysphagia and risk of aspiration. This review documents some proven correlation with declining functional status and dysphagia in PD and ALS. However, correlation over time in longitudinal studies, and extension of this concept to other diseases such as MS and HD was not found in the existing literature. A more cohesive prospective approach at recognizing dysphagia in this important population is warranted

\section{Acknowledgments}

None.

\section{Conflicts of interest}

Author declares there are no conflicts of interest.

\section{Funding}

None.

\section{References}

1. Isaac CA, Franceschi A. Evidence-based medicine: how to practice and teach EBM. Toronto, Ontario: Churchill. 2008;14(15):659-659.

2. Carneiro D, das Graças Wanderley de Sales Coriolano M, Belo LR, de Marcos Rabelo AR, et al. Quality of life related to swallowing in Parkinson's disease. Dysphagia. 2014;29(5):578-582.

3. Guo X, Song W, Chen K, et al. Disease duration-related differences in non-motor symptoms: a study of 616 Chinese Parkinson's disease patients. J Neurol Sci. 2013;330(1-2):32-37.

4. Walker RW, Dunn JR, Gray WK. Self-reported dysphagia and its correlates within a prevalent population of people with Parkinson's disease. Dysphagia. 2011;26(1):92-96.

5. Leow LP, Huckabee ML, Anderson T, et al. The impact of dysphagia on quality of life in ageing and Parkinson's disease as measured by the swallowing quality of life (SWAL-QOL) questionnaire. Dysphagia. 2010;25(3):216-220.

6. Plowman-Prine EK, Sapienza CM, Okun MS, et al. The relationship between quality of life and swallowing in Parkinson's disease. Mov Disord. 2009;24(9):1352-1358.

7. Lorefält B, Granérus AK, Unosson M. Avoidance of solid food in weight losing older patients with Parkinson's disease. J Clin Nurs. 2006;15(11):1404-1412.

8. Müller J, Wenning GK, Verny M, et al. Progression of dysarthria and dysphagia in postmortem-confirmed parkinsonian disorders. Arch Neurol. 2001;58(2):259-264.

9. Miller N, Allcock L, Hildreth AJ, et al. Swallowing problems in Parkinson disease: frequency and clinical correlates. J Neurol Neurosurg Psychiatry. 2009;80(9):1047-1049.
10. Antonini A, Barone P, Marconi R, et al. The progression of non-motor symptoms in Parkinson's disease and their contribution to motor disability and quality of life. J Neurol. 2012;259(12):2621-2631.

11. Auyeung M, Tsoi TH, Mok V, et al. Ten year survival and outcomes in a prospective cohort of new onset Chinese Parkinson's disease patients. $J$ Neurol Neurosurg Psychiatry. 2012;83(6):607-611.

12. Volonté MA, Porta M, Comi G. Clinical assessment of dysphagia in early phases of Parkinson's disease. Neurol Sci. 2002;23(Suppl 2):S121-S122.

13. Potulska A, Friedman A, Królicki L, et al. Swallowing disorders in Parkinson's disease. Parkinsonism Relat Disord. 2003;9(6):349-353.

14. Monte FS, da Silva-Júnior FP, Braga-Neto P, et al. Swallowing abnormalities and dyskinesia in Parkinson's disease. Mov Disord. 2005;20(4):457-462.

15. Chiò A, Hammond ER, Mora G, et al. Development and evaluation of a clinical staging system for amyotrophic lateral sclerosis. J Neurol Neurosurg Psychiatry. 2005;86(1):38-44.

16. Nakamura R, Atsuta N, Watanabe H, et al. Neck weakness is a potent prognostic factor in sporadic amyotrophic lateral sclerosis patients. $J$ Neurol Neurosurg Psychiatry. 2013;84(12):1365-1371.

17. Magnus $\mathrm{T}$, Beck M, Giess $\mathrm{R}$, et al. Disease progression in amyotrophic lateral sclerosis: predictors of survival. Muscle Nerve. 2002;25(5):709-714.

18. Chiò A1, Mora G, Leone M, et al. Early symptom progression rate is related to ALS outcome: a prospective population-based study. Neurology. 2002;59(1):99-103.

19. del Aguila MA, Longstreth WT, McGuire V, et al. Prognosis in amyotrophic lateral sclerosis: a population-based study. Neurology. 2003;60(5):813-819.

20. Hoehn MM, Yahr MD. Parkinsonism: onset, progression, and mortality. 1967. Neurology. 2001;57(10 Suppl 3):S11-S26.

21. Hadjikoutis S, Wiles CM. Respiratory complications related to bulbar dysfunction in motor neuron disease. Acta Neurol Scand. 2001;103(4):207-213.

22. Altman KW, Richards A, Goldberg L, et al. Dysphagia in stroke, neurodegenerative disease, and advanced dementia. Otolaryngol Clin North Am. 2013;46(6):1137-1149.

23. Altman KW. Understanding dysphagia: a rapidly emerging problem. Otolaryngol Clin North Am. 2013;46(6): xiii-xvi.

24. Rosenbek JC, Robbins JA, Roecker EB, et al. A penetration-aspiration scale. Dysphagia. 1996;11(2):93-98.

25. McHorney CA, Robbins J, Lomax K, et al. The SWAL-QOL and SWAL-CARE outcomes tool for oropharyngeal dysphagia in adults: III. Documentation of reliability and validity. Dysphagia . 2002;17(2):97-114.

26. Waxman MJ, Durfee D, Moore M, et al. Nutritional aspects and swallowing function of patients with Parkinson's disease. Nutr Clin Pract. 1990;5(5):196-199.

27. Kennedy G, Pring T, Fawcus R. No place for motor speech acts in the assessment of dysphagia? Intelligibility and swallowing difficulties in stroke and Parkinson's disease patients. Eur J Disord Commun. 1993;28(2):213-226.

28. Speyer R. Oropharyngeal dysphagia: screening and assessment. Otolaryngol Clin North Am. 2013;46(6):989-1008.

29. Ramaker C, Marinus J, Stiggelbout AM, et al. Systematic evaluation of rating scales for impairment and disability in Parkinson's disease. Mov Disord. 2002;17(5):867-876. 
30. Zigmond A, Snaith R. The hospital anxiety and depression scale. Acta Neurol Scand. 1983;67(6):361-370.

31. Dubois B, Slachevsky A, Litvan I, et al. The FAB: a Frontal Assessment Battery at bedside. Neurology. 2000;55(11):1621-1626.

32. Hamilton M. A rating scale for depression. J Neurol Neurosurg Psychiatry. 1960;23:56-62.

33. Voustianiouk A, Seidel G, Panchal J, et al. ALSFRS and appel ALS scores: discordance with disease progression. Muscle Nerve. 2008;37(5):668-672.
34. Appel V, Stewart SS, Smith G, et al. A rating scale for amyotrophic lateral sclerosis: description and preliminary experience. Ann Neurol. 1987;22(3):328-333.

35. Cedarbaum JM, Stambler N, Malta E, et al. The ALSFRS-R: a revised ALS functional rating scale that incorporates assessments of respiratory function. BDNF ALS Study Group (Phase III). J Neurol Sci. 1999;169(1-2):13-21.

36. Weintraub D, Oehlberg KA, Katz IR, et al. Test characteristics of the 15item geriatric depression scale and Hamilton Depression Rating Scale in Parkinson disease. Am J Geriatr Psychiatry. 2006;14(2):169-175. 